\title{
Enhancement of depsipeptide-mediated apoptosis of lung or esophageal cancer cells by flavopiridol: Activation of the mitochondria-dependent death-signaling pathway
}

Dao M. Nguyen, MD, FRCSC, FACS

William D. Schrump*

Wilson S. Tsai, MD

Aaron Chen, MS

John H. Stewart IV, MD

Federico Steiner, MD

David S. Schrump, MD, FACS

From the Section of Thoracic Oncology, Surgery Branch, Center for Cancer Research, National Cancer Institute, National Institutes of Health, Bethesda, Md.

Read at the Eighty-second Annual Meeting of The American Association for Thoracic Surgery, Washington, DC, May 5-8, 2002.

Received for publication May 30, 2002; accepted for publication Sept 9, 2002.

Address for reprints: Dao M. Nguyen, MD, Senior Investigator, Section of Thoracic Oncology, Surgery Branch, Center for Cancer Research, National Cancer Institute, National Institutes of Health, 10 Center Dr, Room 2B07, Bethesda, MD 20892 (E-mail: Dao_Nguyen@nih.gov).

*Recipient, Cancer Research Training Award, National Cancer Institute.

J Thorac Cardiovasc Surg 2003;125: $1132-42$

doi: $10.1067 / \mathrm{mtc} .2003 .180$
Objective: Treating cancer cells with depsipeptide, a novel antitumor agent currently in a phase II clinical trial, causes potent upregulation of p21/WAF1 expression and cell arrest at G1 and G2 checkpoints. p21/WAF1 upregulation, however, impedes the ability of depsipeptide to induce significant apoptosis. This study was designed to determine whether flavopiridol, a synthetic cyclindependent kinase inhibitor known to inhibit p21 expression in tumor cells, could enhance depsipeptide-mediated apoptosis in cultured lung and esophageal cancer cells.

Methods: Lung or esophageal cancer cells were exposed to depsipeptide, flavopiridol, or a combination of depsipeptide and flavopiridol. Cytotoxicity and apoptosis were quantitated by means of (4,5-dimethylthiazo-2-yl)-2,5-diphenyl tetrazolium bromide and terminal deoxynucleotidyltransferase-mediated dUTP nick end labeling-based assays, respectively. Cytosolic cytochrome c levels, caspase 9 activity, mitochondrial membrane depolarization, and dependence of apoptosis on caspase 9 in treated cells were studied to determine the role of the mitochondria in mediating apoptosis induced by this drug combination.

Results: Flavopiridol completely abolished depsipeptide-mediated dose-dependent upregulation of p21/WAF1 expression. Combining flavopiridol with depsipeptide resulted in a 3- to 8-fold reduction of depsipeptide inhibitory concentration of $50 \%$ values that was closely paralleled by synergistic enhancement of apoptosis (4- to 10-fold higher than levels of cell death induced by either drug alone) in all cancer cell lines. The essential role of mitochondria in mediating cell death was indicated by robust translocation of cytochrome $\mathrm{c}$ from the mitochondria into the cytosol, 2.5- to 5-fold activation of caspase 9, severe disruption of mitochondrial inner membrane potential, and complete inhibition of apoptosis by the selective caspase 9 inhibitor. More important, this drug combination was not toxic to primary normal epithelial cells derived from the airway or skin.

Conclusion: The depsipeptide plus flavopiridol combination exhibits powerful and selective cytocidal activity against cancer but not normal cells. Apoptosis induced by this combination is mediated by the mitochondria-dependent death pathway. 


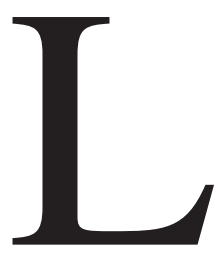

ocally advanced or metastatic non-small cell lung cancer (NSCLC) and esophageal cancer $(\mathrm{EsC})$ are generally refractory to current state-of-the-art multimodality therapies. Indeed, there has been little progress in the treatment outcomes for patients with metastatic NSCLC. ${ }^{1}$ The disappointing results of current therapy for advanced lung cancer or EsC in conjunction with increasing appreciation of the molecular basis of carcinogenesis provide the impetus for ongoing efforts to develop novel and potentially more effective targeted molecular therapeutic strategies for cancers of the aerodigestive tract.

Depsipeptide, a bicyclic polypeptide isolated from the fermentation broth of Chromobacterium violaceum, belongs to a new class of antitumor agents known as histone deacetylase (HDAC) inhibitors. This compound exhibited cytotoxicity against a wide range of cancers in preclinical studies, ${ }^{2}$ and it is currently in a phase II evaluation at the National Cancer Institute. Depsipeptide, similar to other HDAC inhibitors, mediates powerful cell-cycle arrest at G0/G1 and G2/M checkpoints. ${ }^{3-5}$ This is attributable in part to its ability to upregulate the expression of the cyclindependent kinase (cdk) inhibitor p21/WAF1. This druginduced p21 upregulation, although effective in causing cell-cycle arrest, has been shown to impede the ability of depsipeptide and other HDAC inhibitors to mediate programmed cell death. ${ }^{5}$ Abrogation of depsipeptide-mediated p21 upregulation was shown to enhance its ability to induce apoptosis. ${ }^{5}$

Flavopiridol is a synthetic cdk inhibitor that is currently in early clinical development. We have previously demonstrated that flavopiridol mediates apoptosis in EsC cells. ${ }^{6}$ Moreover, flavopiridol has been shown to transciptionally inhibit p21/WAF1 expression in U937 leukemia cells in more recent studies. ${ }^{7,8} \mathrm{We}$ therefore hypothesized that flavopiridol might abrogate depsipeptide-mediated upregulation of p21/WAF1 expression, thereby enhancing apoptosis in NSCLC and EsC cells.

\section{Materials and Methods}

\section{Cells and Reagents}

The NSCLC cells H322 and H460 and the EsC cells TE-12 and TE-13 were maintained in RPMI-1640 culture medium supplemented with glutamine $(2 \mathrm{mmol} / \mathrm{L})$, penicillin $(100 \mathrm{U} / \mathrm{mL})$, streptomycin $(100 \mu \mathrm{g} / \mathrm{mL})$, and $10 \%$ fetal calf serum (RPMI complete). Primary normal human bronchial epithelial cells and normal human epidermal keratinocytes were purchased from Clonetics Corp (Walkerville, $\mathrm{Md}$ ) and grown in serum-free culture media as per the vendor's instructions. Depsipeptide and flavopiridol (obtained from the Cancer Treatment and Evaluation Program, National Cancer Institute, Bethesda, Md) were dissolved in dimethyl sulfoxide as $250 \mathrm{mg} / \mathrm{mL}$ and $2 \mathrm{mmol} / \mathrm{L}$ stocks, respectively, and stored at $-20^{\circ} \mathrm{C}$. The caspase 9 functional assay kit and the cytochrome c enzyme-linked immunosorbent assay (ELISA) kit

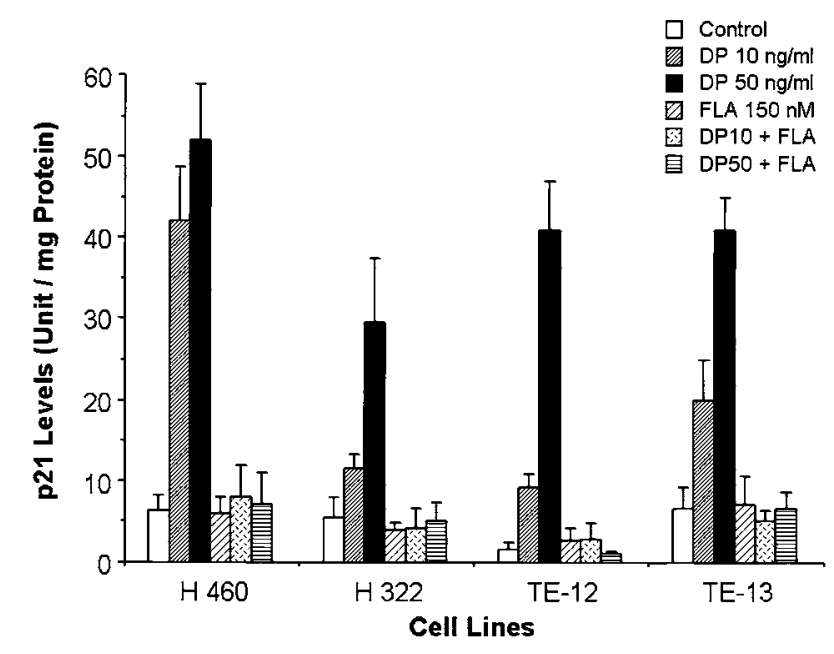

Figure 1. Abrogation of depsipeptide (DP)-mediated upregulation of p21 protein expression in NSCLC or EsC cells by flavopiridol (FLA). Values are means \pm SD of 4 independent experiments.

were purchased from R\&D Systems (Minneapolis, Minn). JC-1 dye and (4,5-dimethylthiazo-2-yl)-2,5-diphenyl tetrazolium bromide (MTT) were obtained from Molecular Probes (Eugene, Ore) and Sigma (St Louis, Mo), respectively.

\section{Cytotoxic Assays}

Cells were seeded into 96-well microtiter plates and incubated overnight before drug treatment. For the dose-dependent cytotoxicity assay, cells were treated with depsipeptide (1-50 ng/mL) for 6 hours. After drug removal and one wash with drug-free RPMI complete medium, cells were further treated with flavopiridol (50 or $100 \mathrm{nmol} / \mathrm{L}$ ) for 66 hours. Viable cells were quantitated by using the MTT assay. For time-course experiments, cells were similarly treated with depsipeptide, flavopiridol, or a combination of depsipeptide and flavopiridol, and cell viability was determined at 24,48 , and 72 hours after the onset of drug exposure. The sensitivity of tumor cells to depsipeptide (after depsipeptide treatment alone or in combination with flavopiridol) was determined by estimating the depsipeptide doses that induced 50\% growth inhibition from the dose-response curves (appropriate corrections were made to account for the mild growth-inhibitory effect of flavopiridol when calculating depsipeptide doses that induced 50\% growth inhibition of cells treated with the drug combination). Drug-induced apoptosis was assessed by using a terminal deoxynucleotidytransferase-mediated dUTP nick end labeling (TUNEL)-based Apo-BrdU assay with a commercially available kit (Pharmingen, Torrence, Calif).

\section{Quantitation of p21/WAF1}

Cells were harvested 24 hours after the onset of depsipeptide, flavopiridol, or depsipeptide plus flavopiridol treatments, and p21/ WAF1 levels were measured by means of ELISA (Oncogene Science, Boston, Mass), normalized for protein content of cell lysates, and expressed as units per milligram of cellular protein. 

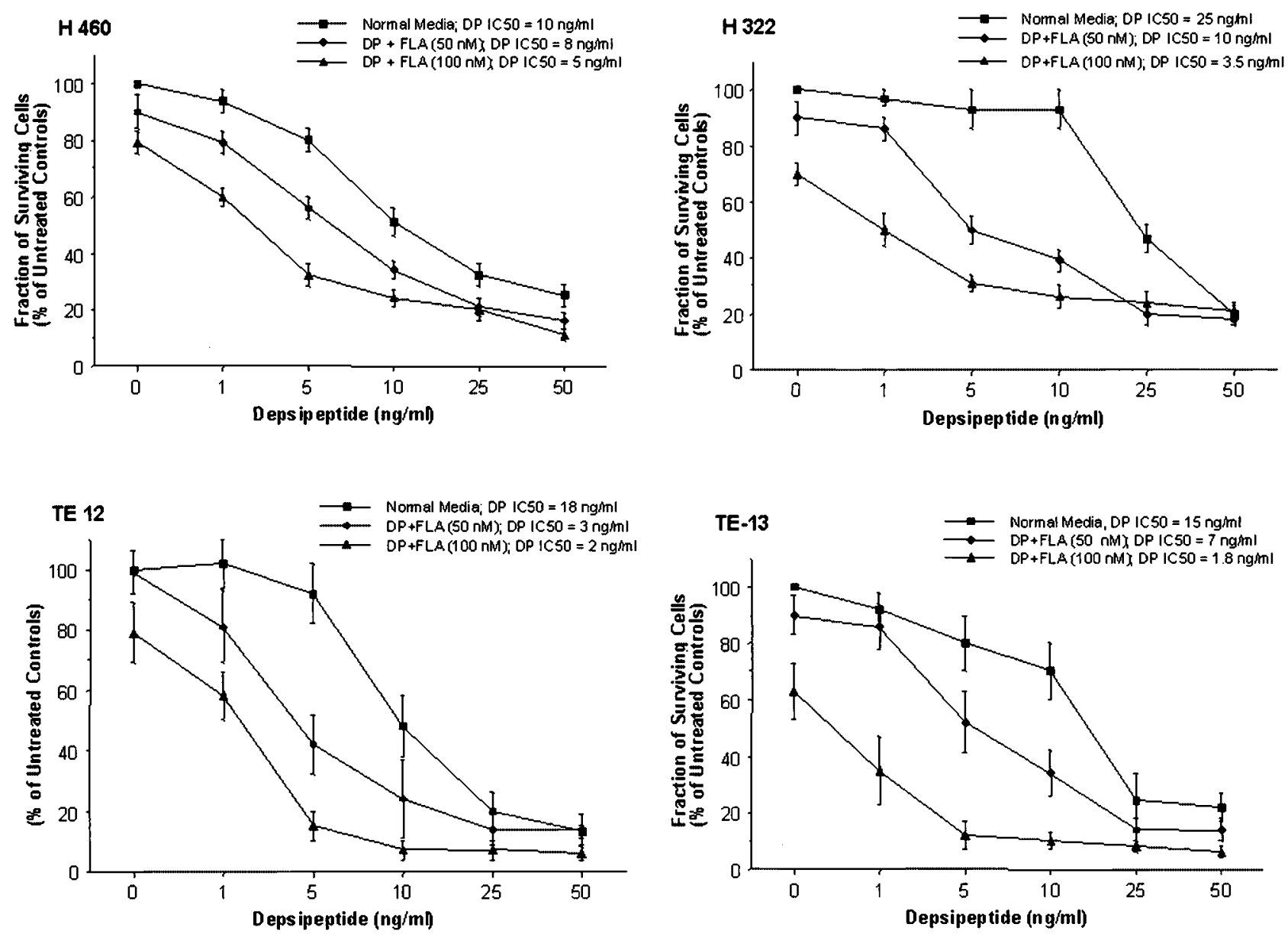

Figure 2A. Dose-dependent enhancement of depsipeptide (DP)-mediated cytotoxicity of the NSCLC cells H460 and H322 and the EsC cells TE-12 and TE-13 by flavopiridol (FLA; $50-100 \mathrm{nmol} / \mathrm{L}$ ). The sensitivity of tumor cells to depsipeptide is indicated by depsipeptide doses that induced $50 \%$ growth inhibition (IC50, in nanograms per milliliter). Values shown are means \pm SD of 4 independent experiments.

Measurements of Caspase 9 Activity and Cytosolic Cytochrome c

Cytosolic extracts were prepared from whole-cell lysates by using commercially available reagents and protocols (Clontech, Palo Alto, Calif). Caspase 9 activity and cytosolic cytochrome c levels in cells treated with depsipeptide, flavopiridol, or depsipeptide plus flavopiridol were determined by using a functional assay kit and by an using ELISA, respectively. Cytochrome $\mathrm{c}$ and caspase 9 levels were corrected for protein content of the samples and expressed as fold increases over those of untreated control samples.

\section{Determination of Mitochondrial Inner Membrane \\ Potential}

Cells were harvested by means of trypsinization 36 hours after the onset of drug treatment and washed once with drug-free culture medium. The cells were then incubated with JC-1 for 30 minutes at $37^{\circ} \mathrm{C}$. Cells were washed twice with phosphate-buffered saline solution and immediately analyzed by means of flow cytometry.
Loss of mitochondrial inner membrane potential was indicated by reduction of JC-1 red fluorescence.

\section{Statistical Analysis}

Data are presented as means \pm SD. Statistical analysis, when indicated, was performed by using the Student $t$ test.

\section{Results}

Abrogation of Depsipeptide-Mediated Upregulation of p21 by Flavopiridol

Exposure of H460 and H322 NSCLC cells or TE-12 and TE-13 EsC cells to depsipeptide for 6 hours resulted in a dose-dependent increase of p21/WAF1 expression ranging from 6- to 10-fold of respective baseline levels. Flavopiridol treatment $(150 \mathrm{nmol} / \mathrm{L})$, although not affecting the basal 

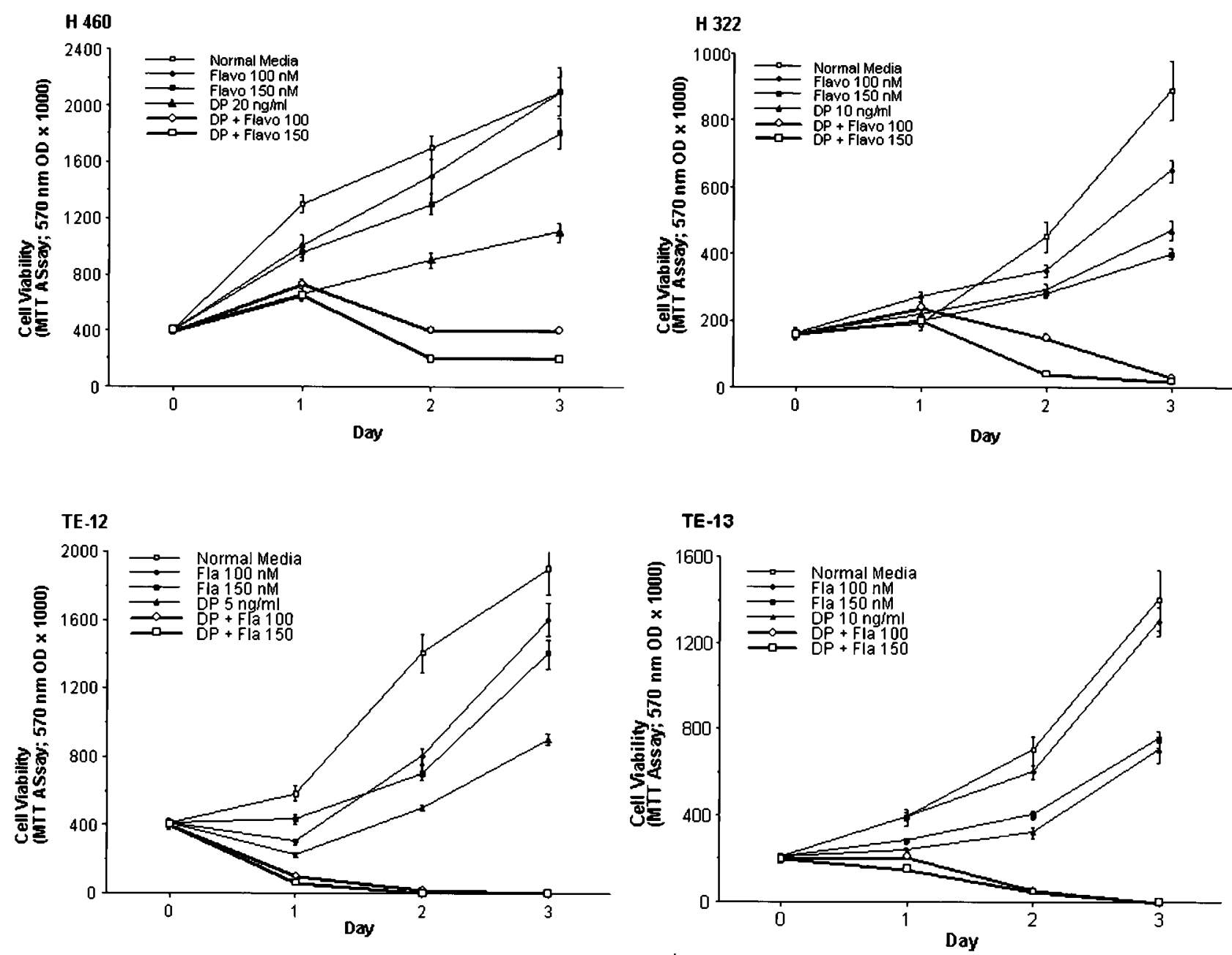

Figure 2B. Time course of the synergistic cytocidal effect of the depsipeptide (DP) plus flavopiridol (FLA) drug combination in H460, H322, TE-12, and TE-13 cells. Although individual depsipeptide or flavopiridol treatment resulted in retardation of cell proliferation, significant cell death was observed in cells treated with the drug combination. Cell viability was quantitated by means of MTT assay and expressed as an optical density (OD) at $570 \mathrm{~nm}$. Values shown are means \pm SD of 3 independent experiments.

p21/WAF1 expression, completely abrogated the depsipeptide-mediated upregulation of this cdk inhibitor (Figure 1).

Synergistic Cytotoxic Effect of the Depsipeptide Plus Flavopiridol Combination in Cancer Cell Lines but not Primary Normal Cells

Exposure of tumor cells to depsipeptide for 6 hours resulted in a dose-dependent inhibition of cell growth. Subsequent treatment of depsipeptide-exposed tumor cells with flavopiridol $(50-100 \mathrm{nmol} / \mathrm{L})$ led to drastic dose-dependent inhibition of cell proliferation that greatly exceeded the mild growth-inhibitory effect of flavopiridol noted in tumor cells treated with this agent alone (Figure 2A). The synergistic cytotoxic effect of this drug combination was most pro- nounced in H322, TE-12, and TE-13 cells. Time-course experiments indicated that although tumor cells treated with either depsipeptide alone or flavopiridol alone exhibited varying degrees of growth retardation (Figure 2B), active cell death occurred in those treated with the drug combination (evidenced by rapid reduction of viable cells to levels well below those present at the beginning of the experiment). In TE-12 or TE-13 cells the cytotoxic effect of this drug combination was so powerful that all tumor cells were eliminated by 48 hours after the onset of drug exposure.

To determine whether the depsipeptide plus flavopiridol combination would be toxic to normal cells, similar experiments were conducted with primary normal cells (human epidermal keratinocytes or bronchial epithelial cells) grown 

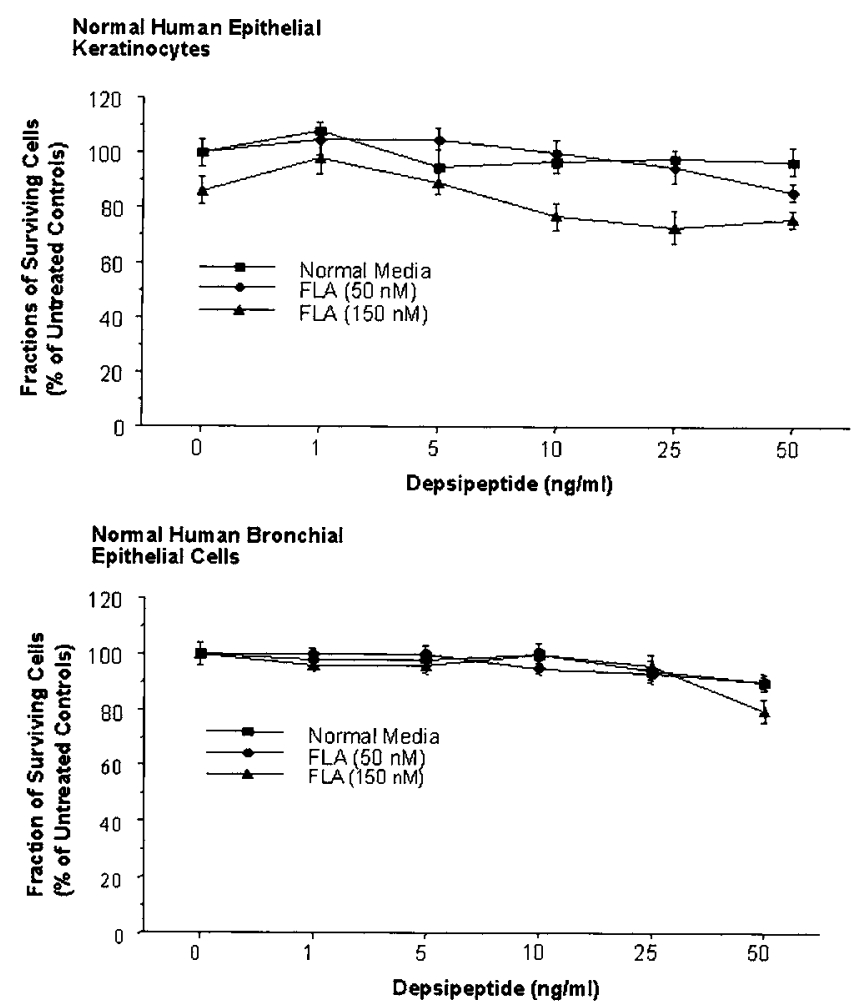

Figure 2C. Lack of significant cytotoxic effect of depsipeptide, flavopiridol (FLA), or the depsipeptide plus flavopiridol combination in normal cells. Values shown are means \pm SD of 3 independent experiments.

to confluence in vitro. Quite remarkably, this drug combination, although being extremely powerful in killing cancer cells, demonstrated only marginal cytotoxic effects toward normal cells (Figure 2C).

Massive induction of apoptosis was noted in all tumor cells treated with this drug combination by using the TUNEL-based Apo-BrdU assay to detect DNA fragmentation. For instance, although $15 \%$ and $20 \%$ of apoptotic cells were observed after depsipeptide or flavopiridol treatment in TE-12 or TE-13 esophageal cells, up to $90 \%$ of these cells were apoptotic within 48 hours after exposure to the sequential depsipeptide plus flavopiridol combination, a clear synergistic combination effect (Figure 3). Similar observations were made when the NSCLC cells H460 or H322 were treated with depsipeptide, flavopiridol, or the depsipeptide plus flavopiridol combination, albeit at a slightly lower magnitude of apoptosis (Figure 3).

The Depsipeptide Plus Flavopiridol Combination Induced Apoptosis Through the MitochondriaDependent Pathway

Expression of Bax (proapoptosis) and Bcl2 (antiapoptosis) proteins in tumor cells (H322 and TE-12 cells) treated with

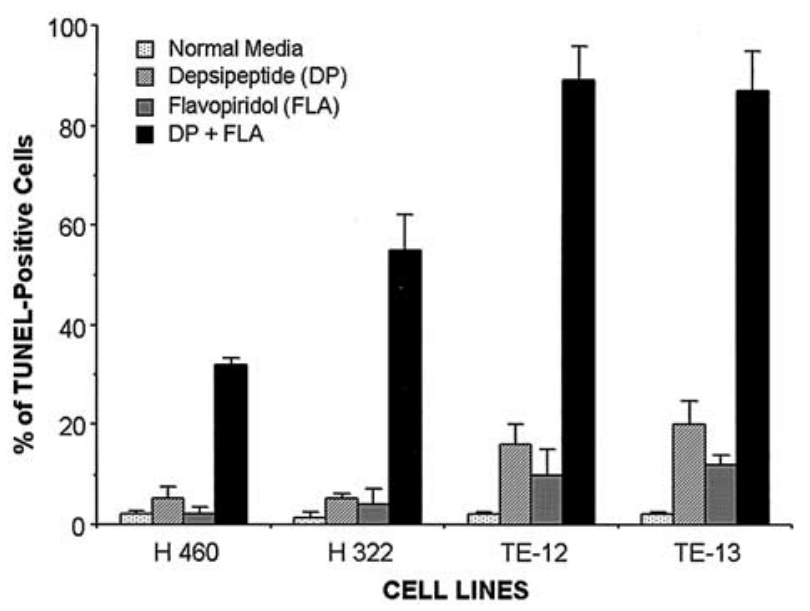

Figure 3. Profound and synergistic induction of apoptosis in NSCLC or EsC cells by the depsipeptide (DP) plus flavopiridol (FLA) drug combination. Values shown are means $\pm S D$ of 3 independent experiments.

depsipeptide, flavopiridol, or the depsipeptide plus flavopiridol combination was determined by Western blotting, and their relative levels were quantitated by densitometry. In H322 cells depsipeptide plus flavopiridol treatment was associated with a strong upregulation of Bax and unchanged Bcl 2 levels. On the other hand, the Bax level remained unchanged, whereas the Bcl2 level decreased after depsipeptide plus flavopiridol in TE-12 cells. The net result in both cases was a profound increase in $\mathrm{Bax} / \mathrm{Bcl} 2$ ratios that favors the induction of apoptosis ${ }^{9}$ in cells treated with the depsipeptide plus flavopiridol combination (Figure 4).

In conjunction with alterations of $\mathrm{Bax}$ and $\mathrm{Bcl} 2$ levels was the massive release of cytochrome c from the mitochondrial intermembrane space into the cytosol in NSCLC and EsC cells treated with the depsipeptide plus flavopiridol combination (Figure 5). Once released from mitochondria into the cytoplasm, cytochrome c combines with Apaf-1, adenosine triphosphate, and procaspase 9 to form the apotosome, which catalytically activates caspase 9 and thus initiates caspase-dependent programmed cell death. ${ }^{10} \mathrm{Al}-$ though cancer cells treated with either depsipeptide or flavopiridol treatment alone had minimal effect on caspase 9 activity, those treated with the depsipeptide plus flavopiridol combination exhibited a 2.8- to 5-fold increase in the levels of caspase 9 activity starting around 24 hours (H322, TE-12, and TE-13) to 36 hours (H460) after the onset of drug exposure (Figure 6). Moreover, depsipeptide plus flavopiridol treatment of cancer cells led to profound mitochondrial damage indicated by massive loss of mitochondria inner membrane potentials (depolarization), as detected by means of JC-1 staining and flow cytometry (Figure 7). Cells with depolarized mitochondria exhibited reduction of red but not green fluorescence and were gated to the right 

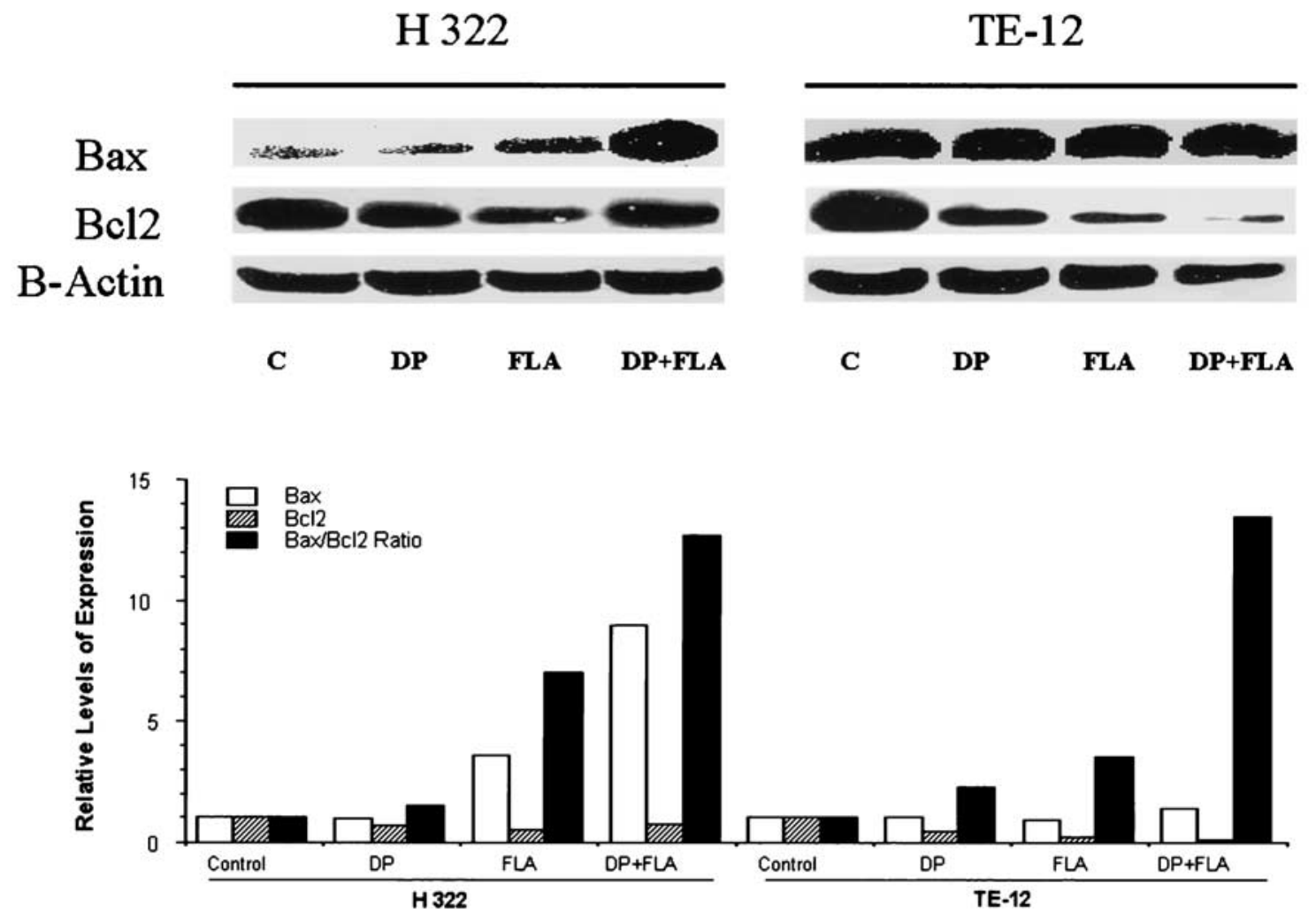

Figure 4. Western blot analysis of drug-induced alteration of Bax and Bcl2 levels in H322 or TE-12 cells at 18 hours after the onset of drug exposure. The relative levels of Bax and Bcl2 were quantitated by densitometry. This finding was reproduced in another independent experiment. DP, Depsipeptide; FLA, flavopiridol.

lower quadrants of the dot plots. The robust apoptosis mediated by the depsipeptide plus flavopiridol combination was almost completely abrogated by the selective caspase 9 inhibitor, conclusively affirming the notion that mitochondria-dependent caspase 9 activation was essential for depsipeptide plus flavopiridol-mediated apoptosis (Figure 8).

\section{Discussion}

Depsipeptide is currently under phase II evaluation in patients with refractory lung cancers at the National Cancer Institute. The pharmacokinetic and toxicity profiles of depsipeptide derived from the phase I trial have been recently reported. ${ }^{11}$ Depsipeptide has been shown to deplete multiple growth signaling-related proteins (mutant p53, erbB1, erbB2, and Raf-1), as well as to alter the expression of several cell-cycle proteins (upregulation of p21/WAF1 and cyclin E, downregulation of cyclin A) in lung cancer cells, all of which might contribute to the antiproliferative effect of this agent. ${ }^{12}$ Modest induction of apoptosis was observed after depsipeptide treatment but only after 24 hours of drug exposure. ${ }^{12}$ Upregulation of $\mathrm{p} 21 / \mathrm{WAF} 1$ is a constant feature of HDAC inhibitors, including depsipeptide. Although responsible for cell arrest at G1 and G2 checkpoints, it actu-

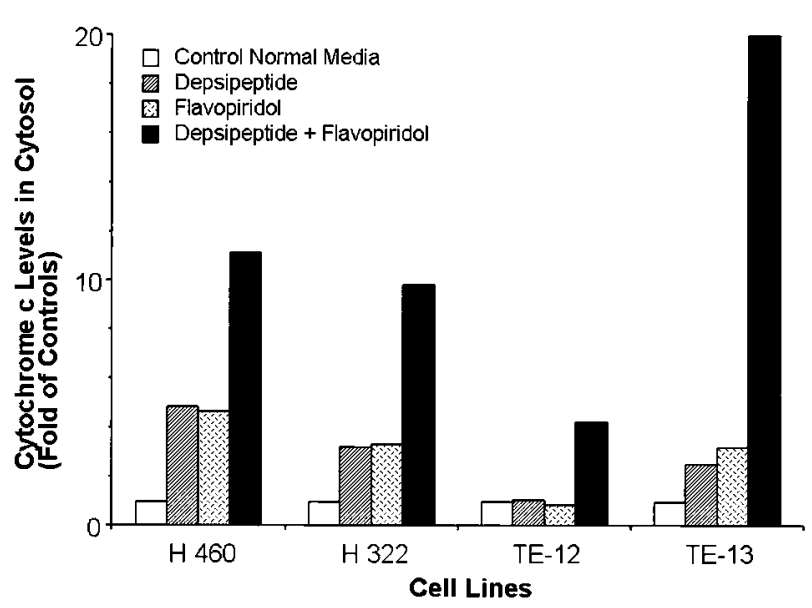

Figure 5. Significant accumulation of cytochrome $\mathrm{c}$ in the cytosolic fraction of the cell lysates was noted in NSCLC H460 and H322 cells or EsC TE-12 and TE-13 cells after treatment with the depsipeptide plus flavopiridol drug combination. Cells were harvested at 20 hours (H322, TE-12, and TE-13 cells) or 40 hours (H460) after the onset of drug exposure; cytosolic cytochrome c levels were measured by ELISA. Representative data from a series of 3 independent experiments with similar results are shown. 

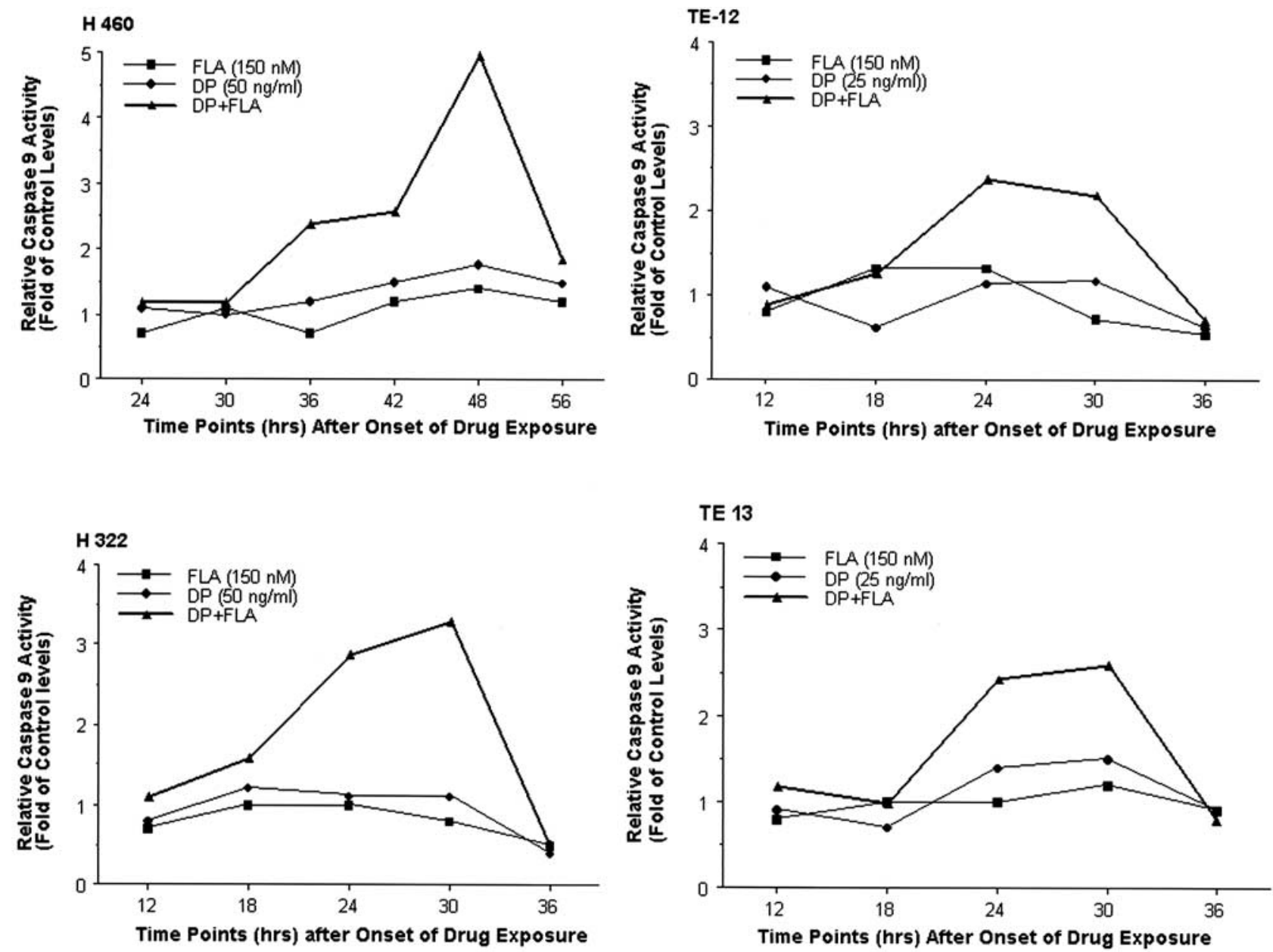

Figure 6. The time course of caspase 9 activation in cancer cell lines after depsipeptide (DP), flavopiridol (FLA), or depsipeptide plus flavopiridol treatments. Relative levels of caspase 9 activities are shown as folds of levels in untreated control cells.

ally reduces cytotoxicity and apoptosis mediated by these agents, as suggested by many lines of evidence: p21-/cells were more susceptible to apoptosis induced by HDAC inhibitors than their $\mathrm{p} 21+/+$ parental cells, 3,5 interference with $\mathrm{p} 21 / \mathrm{WAF} 1$ upregulation by a p21 antisense construct enhances the cytocidal effect of HDAC inhibitors, ${ }^{8}$ and cells lacking the usual p21/WAF1 upregulation response are very sensitive to the cytotoxic effect of HDAC inhibitors. ${ }^{5}$ The logical approach one would take to augment the therapeutic efficacy of depsipeptide or other HDAC inhibitors in addition to using higher doses or longer durations of drug exposure (associated with higher propensity for side effects) is to inhibit drug-induced $\mathrm{p} 21 / \mathrm{WAF} 1$ upregulation by using either an approved drug or an experimental agent that is currently under clinical development. The resulting combination should also be clinically applicable and safe. Flavopiridol, a potent cdk inhibitor, appeared to fit this role very well. On the basis of its favorable antitumor activity observed in preclinical studies, flavopiridol has been used in clinical trials to treat solid tumors, either as single agent or in combination with conventional chemotherapeutics. ${ }^{13,14}$ The pharmacokinetic and toxicity profiles of this agent have been well described in phase I and II clinical trials. ${ }^{13,14}$ As a single agent, flavopiridol was been reported to modulate the expression of $\mathrm{Bax}, \mathrm{Bcl} 2$, or both and to induce apoptosis of cancer cells in vitro. ${ }^{6,14,15}$ Of particular relevance to our study is the finding that flavopiridol transcriptionally inhibited $\mathrm{p} 21 / \mathrm{WAF} 1$ upregulation mediated by the protein kinase C activator phorbol 12-myristate 13-acetate in U937 leukemia cells. ${ }^{7}$

Our data clearly demonstrate the superior cytotoxic effect of the depsipeptide plus flavopiridol combination to that of either depsipeptide or flavopiridol alone on NSCLC and EsC cells in vitro. The selections of drug treatment schedules (6 hours of depsipeptide and 66 hours of flavopiridol) were based on those used in phase I clinical trials of each 

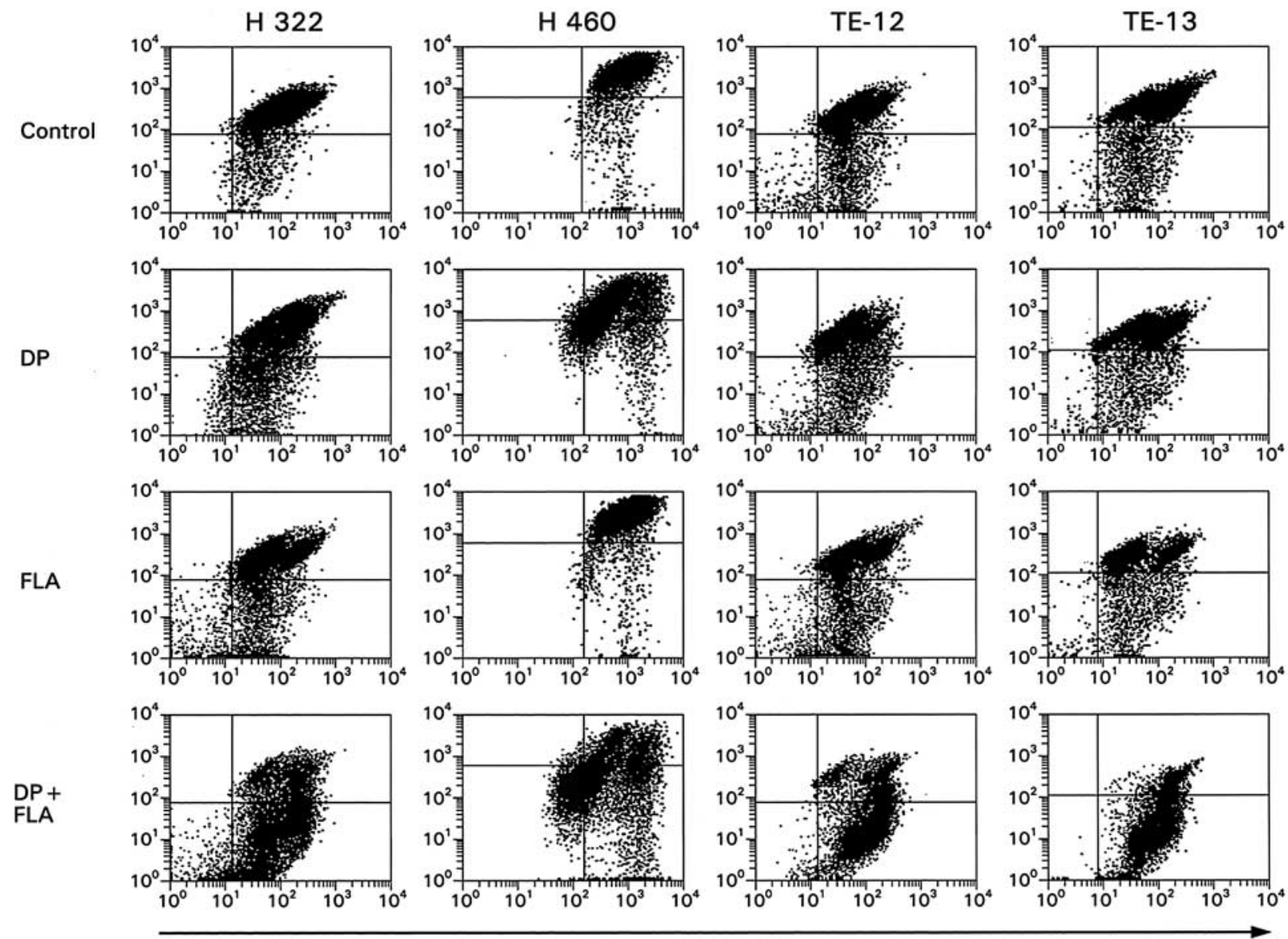

Figure 7. Perturbation of mitochondrial function after drug exposure indicated by the loss of mitochondrial inner membrane potential, as assessed by means of JC-1 staining and flow cytometry. Cells with loss of mitochondrial inner membrane potential are sorted to the right lower quadrant of the dot plots. Representative data from a series of 3 independent experiments with similar results are shown.

respective drug. ${ }^{11,13}$ Moreover, plasma drug concentrations (depsipeptide, $25-50 \mathrm{ng} / \mathrm{mL}$; flavopiridol, 100-150 nmol/L) similar to those required to yield synergistic cytotoxic effects in vitro would be clinically achievable according to pharmacokinetic data obtained in these phase I clinical trials. ${ }^{11,13}$ Another important and clinically relevant finding of our study was the lack of cytotoxicity with this drug combination in primary normal cells grown to confluence in vitro. Normal human bronchial epithelial cells and skin keratinocytes were chosen to represent normal counterparts of NSCLC and EsC cells, respectively. Although depsipeptide or flavopiridol, as single agents, mediate varying degrees of apoptosis in cancer cells derived from solid tumors, $6,11,15,16$ the ability of the drug combination to induce massive apoptosis through a mitochondria-dependent pathway in NSCLC or EsC cells deserves in-depth evaluation.

Mitochondria, although being an indispensable life-supporting component of all eukaryotes, play a crucial role in inducing apoptosis of the very cells they are meant to sustain when appropriately activated by intracellular or extracellular death signals (Figure 9). Extracellular death signals (FasL, tumor necrosis factor, or tumor necrosis factor receptor apoptosis-inducing ligand) bind to their respective receptors to activate caspase 8 , leading to direct or indirect (through activation of Bid, the connector that links extracellular death signals to the mitochondria-dependent death pathway) activation of caspase 3, leading to apoptosis. ${ }^{17}$ Intracellular death signals, such as those initiated with chemotherapeutics or other cytotoxic agents, activate the mitochondria by modulating the function, expression, or both of proapoptotic proteins (eg, Bid, Bax, and Bak), antiapoptotic proteins (eg, Bcl2 and BclXL), or both. ${ }^{18}$ Activated proapoptotic proteins converge on the mitochondrial outer membrane to form conducting channels through homooligomerization or hetero-oligomerization, allowing cytochrome $\mathrm{c}$ that resides in the intermembrane matrix to trans- 


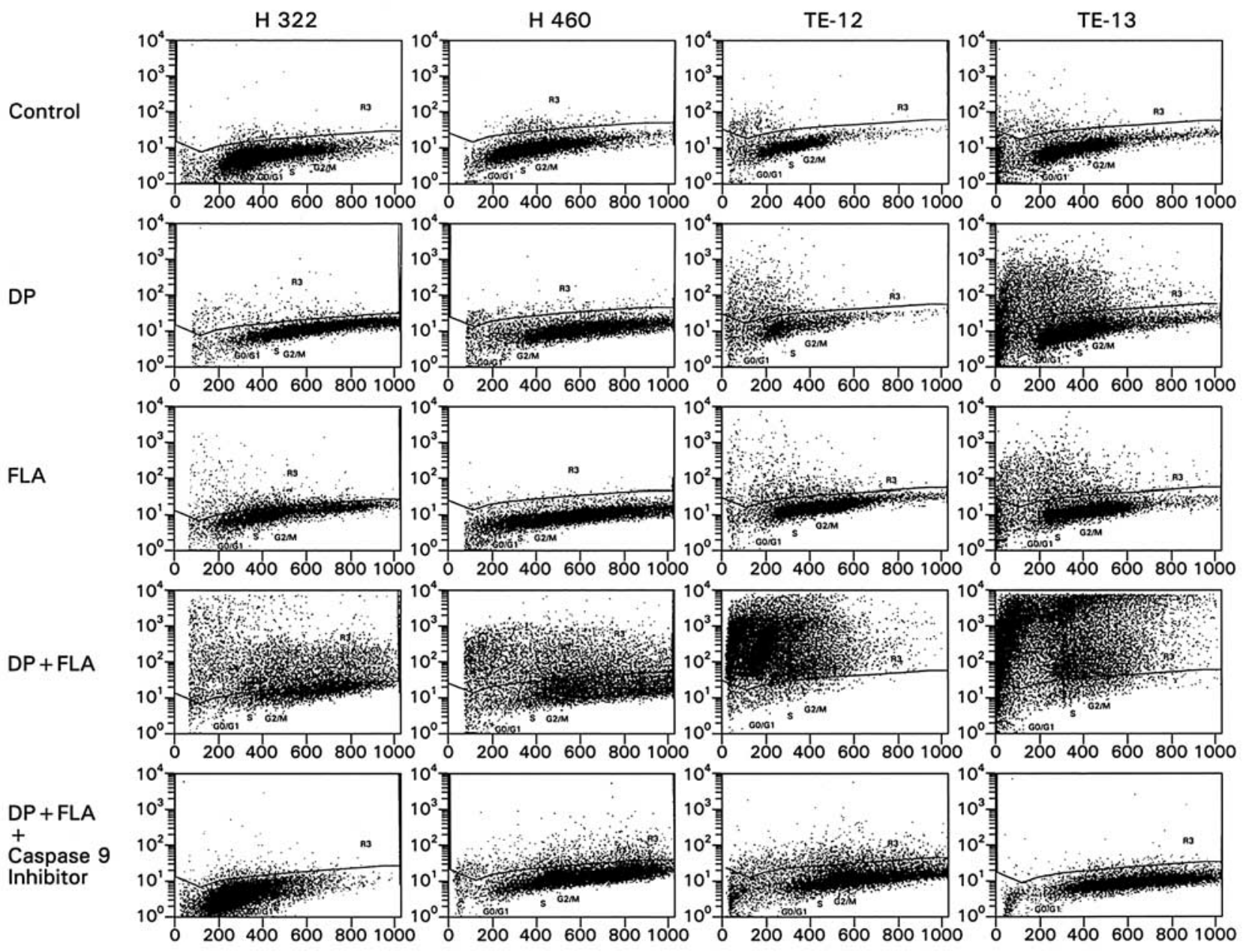

Figure 8. Inhibition of depsipeptide (DP) plus flavopiridol (FLA)-mediated induction of apoptosis in NSCLC cells or EsC cells by the selective caspase 9 inhibitor $(20 \mu \mathrm{mol} / \mathrm{L}$ added to the culture media just before flavopiridol exposure). Representative data from a series of 3 independent experiments with similar results are shown.

locate to the cytoplasm. ${ }^{19}$ Cytosolic cytochrome c, in turn, combines with adenosine triphosphate, procaspase 9, and Apaf-1 to form a complex known as the apoptosome, which activates caspase 9 and, subsequently, caspase $3 .{ }^{10} \mathrm{Bcl} 2$ and BclXL can inhibit the initiation of apoptosis by interfering with the interaction of activated Bax, Bak, and Bid with mitochondria at many levels and thus preventing cytochrome c release. ${ }^{20}$ The depsipeptide plus flavopiridol combination induces mitochondria-dependent apoptosis in tumor cells by targeting the upstream components of this death pathway (ie, the $\mathrm{Bax}$ and $\mathrm{Bcl} 2$ proteins). Indeed, cells treated with this drug combination exhibit alterations of $\mathrm{Bax}$ and $\mathrm{Bcl} 2$ levels associated with the highest $\mathrm{Bax} / \mathrm{Bcl} 2$ ratios that set the appropriate condition for mitochondrial activation, leading to robust translocation of cytochrome $\mathrm{c}$ into the cytosol compartment, caspase 9 activation, and apoptosis. The mechanism by which depsipeptide in combination with flavopiridol alters Bax and $\mathrm{Bcl} 2$ expression is unclear. This and other issues related to the effects of depsipeptide plus flavopiridol on other components upstream and downstream of the mitochondria in this death-signaling pathway are topics of ongoing investigation in our laboratory.

Although not conclusively demonstrated in our present experiments, abrogation of depsipeptide-mediated p21/ WAF1 upregulation by flavopiridol in all likelihood contributes to the profound induction of apoptosis mediated by the sequential depsipeptide plus flavopiridol combination. p21/WAF1 has been demonstrated to bind to procaspase 3 and inhibit its proteolytic activation. ${ }^{21}$ Caspase 3 activation, however, is not an initiating event because it occurs downstream in the caspase-dependent apoptosis cascade. Nevertheless, activated caspase 3 can amplify the apoptosis effect of the upstream death signals by activating caspase 8 through a positive feedback loop. ${ }^{22}$ The complex interaction, 


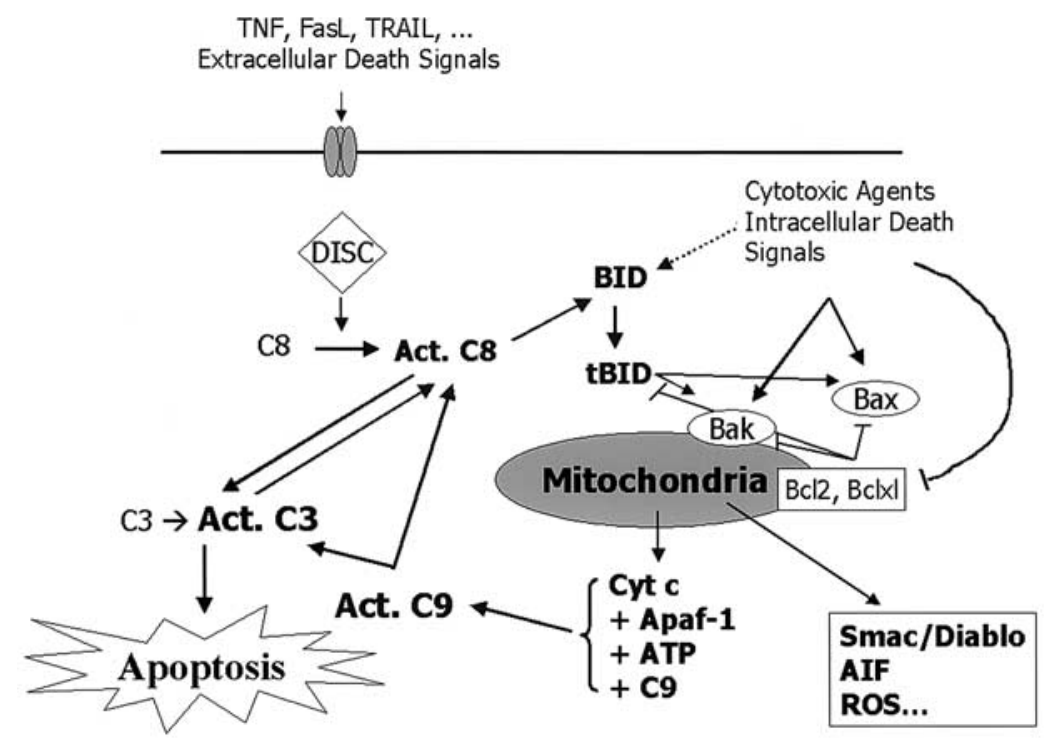

Figure 9. The central role of mitochondria in mediating and amplifying apoptosis initiated by extracellular or intracellular death signals. TNF, Tumor necrosis factor; TRAIL, tumor necrosis factor receptor apoptosis-inducing ligand; DISC, Death-inducing signaling complex; ATP, adenosine triphosphate; Smac/Diablo, second mitochondriaderived activation of caspases; $A I F$, apoptosis-inducing factor; ROS, reactive oxygen species.

connection, or both between p21/WAF1 downregulation and alteration of the $\mathrm{Bax} / \mathrm{Bcl} 2$ ratio observed in cells treated with depsipeptide plus flavopiridol remains to be elucidated.

Although the mechanisms by which sequential depsipeptide plus flavopiridol treatment mediates apoptosis in cancer cells have not been fully elucidated, our data clearly indicate that this novel drug combination exhibits potent cytotoxicity preferentially in malignant and not in normal cells. Preclinical animal studies with human xenografts are being performed to confirm this apparent salutary effect and to evaluate the safety of this drug combination in vivo. These compelling data provide the rationale for further clinical development of this drug combination to treat refractory malignant disease.

We thank Arnold Mixon and Shawn Farid for performing flow cytometric studies.

\section{References}

1. Schiller JH, Harrington D, Belani CP, Langer C, Sandler A, Krook J, et al. Comparison of four chemotherapy regimens for advanced nonsmall-cell lung cancer. N Engl J Med. 2002;346:92-8.

2. Ueda H, Manda T, Matsumoto S, Mukumoto S, Nishigaki F, Kawamura I, Shimomura K. FR901228, a novel antitumor bicyclic depsipeptide produced by Chromobacterium violaceum No 968. Antitumor activities on experimental tumors in mice. J Antibiot. 1994;47:315-23.

3. Sandor V, Senderowicz A, Mertins S, Sackett D, Sausville E, Blagosklonny MV, et al. P21-dependent g(1)arrest with downregulation of cyclin D1 and upregulation of cyclin E by the histone deacetylase inhibitor FR901228. Br J Cancer. 2000;83:817-25.

4. Sambucetti LC, Fischer DD, Zabludoff S, Kwon PO, Chamberlin H, Trogani N, et al. Histone deacetylase inhibition selectively alters the activity and expression of cell cycle proteins leading to specific chromatin acetylation and antiproliferative effects. J Biol Chem. 1999; 274:34940-7.

5. Burgess AJ, Pavey S, Warrener R, Hunter LJ, Piva TJ, Musgrove EA, et al. Up-regulation of p21(WAF1/CIP1) by histone deacetylase inhibitors reduces their cytotoxicity. Mol Pharmacol. 2001; 60:828-37.

6. Schrump DS, Matthews W, Chen GA, Mixon A, Altorki NK. Flavopiridol mediates cell cycle arrest and apoptosis in esophageal cancer cells. Clin Cancer Res. 1998;4:2885-90.

7. Cartee L, Wang Z, Decker RH, Chellappan SP, Fusaro G, Hirsch KG, et al. The cyclin-dependent kinase inhibitor (CDKI) flavopiridol disrupts phorbol 12-myristate 13-acetate-induced differentiation and CDKI expression while enhancing apoptosis in human myeloid leukemia cells. Cancer Res. 2001;61:2583-91.

8. Rosato RR, Wang Z, Gopalkrishnan RV, Fisher PB, Grant S. Evidence of a functional role for the cyclin-dependent kinase-inhibitor p21WAF1/ CIP1/MDA6 in promoting differentiation and preventing mitochondrial dysfunction and apoptosis induced by sodium butyrate in human myelomonocytic leukemia cells (U937). Int J Oncol. 2001;19:181-91.

9. Oltvai ZN, Milliman CL, Korsmeyer SJ. Bcl2 heterodimerizes in vivo with a conserved homolog Bax, that accelerates programmed cell death. Cell. 1993;74:609-19.

10. Li P, Nijhawan D, Budihardjo I, Srinivasula SM, Ahmad M, Alnemri ES, et al. Cytochrome c and dATP-dependent formation of Apaf-1/ caspase-9 complex initiates an apoptotic protease cascade. Cell. 1997; 91:479-89.

11. Sandor V, Bakke S, Robey RW, Kang MH, Blagosklonny MV, Bender J, et al. Phase I trial of the histone deacetylase inhibitor, depsipeptide (FR901228, NSC 630176), in patients with refractory neoplasms. Clin Cancer Res. 2002;8:718-28.

12. Yu X, Guo ZS, Marcu MG, Neckers L, Nguyen DM, Chen GA, Schrump DS. Modulation of p53, ErbB1, ErbB2, and Raf-1 expression in lung cancer cells by depsipeptide FR901228. J Natl Cancer Inst. 2002;94:504-13.

13. Senderowicz AM, Headlee D, Stinson SF, Lush RM, Kalil N, Villalba $\mathrm{L}$, et al. Phase I trial of continuous infusion flavopiridol, a novel cyclin-dependent kinase inhibitor, in patients with refractory neoplasms. J Clin Oncol. 1998;16:2986-99. 
14. Schwartz GK, Ilson D, Saltz L, O'Reilly E, Tong W, Maslak P, et al. Phase II study of the cyclin-dependent kinase inhibitor flavopiridol administered to patients with advanced gastric carcinoma. J Clin Oncol. 2001;19:1985-92.

15. Shapiro GI, Koestner DA, Matranga CB, Rollins BJ. Flavopiridol induces cell cycle arrest and p53-independent apoptosis in non-small cell lung cancer cell lines. Clin Cancer Res. 1999;5:2925-38.

16. Byrd JC, Shinn C, Ravi R, Willis CR, Waselenko JK, Flinn IW, et al. Depsipeptide (FR901228): a novel therapeutic agent with selective, in vitro activity against human B-cell chronic lymphocytic leukemia cells. Blood. 1999;94:1401-8.

17. Scaffidi C, Fulda S, Srinivasan A, Friesen C, Li F, Tomaselli KJ, et al. Two CD95 (APO-1/Fas) signaling pathways. EMBO J. 1998;6:1675-87.

18. Yang E, Korsmeyer SJ. Molecular thanatopsis: a discourse on the $\mathrm{Bcl} 2$ family and cell death. Blood. 1996;88:386-401.

19. Wei MCX, Zong W-X, Cheng EHY, Lindsten T, Panoutsakopoulou V, Ross AJ, et al. Proapoptotic Bax and Bak: a requisite gateway to mitochondria dysfunction and death. Science. 2001;292:727-30.

20. Adams JM, Cory S. The Bcl-2 protein family: arbiters of cell survival. Science. 1998;281:1322-6.

21. Suzuki A, Tsutomi Y, Miura M, Akahanie K. Caspase 3 activation to suppress Fas-mediated apoptosis: identification of a binding domain with p21 and ILP and inactivation machinery by p21. Oncogene. 1999;18:1239-44.
22. Engels H, Stepczynska AQ, Stroh C, Lauber K, Berg C, Schwenzer R, et al. Caspase 8/FLICE functions as an executioner caspase in anticancer drug-induced apoptosis. Oncogene. 2000;19:4563-73.

\section{Discussion}

Dr Stephen G. Swisher (Houston, Tex). Did you use cyclosporine A or bongkrekic acid to try and block the mitochondria, and did that block your apoptosis?

Dr Nguyen. No, I have not tried that. The interesting thing is, when I looked at the time course of cytochrome c release and activation of caspase 9 and the mitochondrial depolarization, there was a dissociation. The depolarization occurred quite late. It happened around 36 hours after onset of drug treatment, whereas cytochrome c release was detected around 18 to 20 hours and activation of caspase 9 was noted around 24 hours after drug exposure. Therefore, I have not tried these blocking agents, but I think membrane depolarization is a product of caspase activation rather than the primary event. 